\title{
Spectroscopic Signatures of Halogens in Clathrate Hydrate Cages. 2. Iodine
}

\author{
Galina Kerenskaya,* Ilya U. Goldschleger, V. Ara Apkarian, Everly Fleischer, and \\ Kenneth C. Janda \\ Department of Chemistry, University of California-Irvine, Irvine, California 92697
}

Received: June 18, 2007; In Final Form: August 14, 2007

\begin{abstract}
UV-vis and Raman spectroscopy were used to study iodine molecules trapped in sII clathrate hydrate structures stabilized by THF, $\mathrm{CH}_{2} \mathrm{Cl}_{2}$, or $\mathrm{CHCl}_{3}$. The spectra show that the environment for iodine inside the water cage is significantly less perturbed than either in aqueous solution or in amorphous water-ice. The resonance Raman progression of $\mathrm{I}_{2}$ in THF clathrate hydrate can be observed up to $v=6$ when excited at $532 \mathrm{~nm}$. The extracted vibrational frequency $\omega_{\mathrm{e}}=214 \pm 1 \mathrm{~cm}^{-1}$ is the same as that of the free molecule to within experimental error. At the same time, the UV-vis absorption spectrum of $\mathrm{I}_{2}$ in the sII hydrate exhibits a relatively large, $1440 \mathrm{~cm}^{-1}$, blue-shift. This is mainly ascribed to the differential solvation of the $\mathrm{I}_{2}$ electronic states. We conclude that iodine in sII hydrate resides in a $5^{12} 6^{4}$ cavity, in which the ground-state $\mathrm{I}_{2}$ potential is not significantly perturbed by the hydrate lattice. In contrast, in water and in ice, the valence absorption band of $I_{2}$ is dramatically broadened and blue-shifted by $3000 \mathrm{~cm}^{-1}$, and the resonance Raman scattering is effectively quenched. These observations are shown to be consistent with a strong interaction between water molecule and iodine through the lone pair of electrons on water as in the case of bromine in the same media. The results presented here, and the stability of other halogen hydrates, were used to test the predictions of simple models and force-field calculations of the host cage-guest association energy.
\end{abstract}

\section{Introduction}

Clathrate hydrates are crystalline inclusion compounds in which the host framework is made of hydrogen bonded water molecules with guest molecules trapped inside the polyhedral cages. Several of the cage types with $\mathrm{Br}_{2}$ inside are shown in Figure 1. Cages of different types combine to form a solid 3-D lattice. The empty lattice is not thermodynamically stable: its free energy is higher than that of hexagonal ice. Therefore, the interaction between the guest molecules and the surrounding water molecules in the clathrate hydrate must be sufficiently stabilizing so that the free energy of the clathrate hydrate is less than that of the separated ice and guest molecules for the hydrate forming temperature and pressure. The host-guest interactions fall in the range of van der Waals effects. Thus, clathrate hydrates are fascinating examples of solids for which the van der Waals forces are crucial for stability.

It has been known for quite some time that the guest size defines the morphology of the clathrate hydrate. ${ }^{1}$ Small atoms and molecules with diameters up to $5.0 \AA$, such as $\mathrm{Ar}, \mathrm{Kr}, \mathrm{N}_{2}$, and $\mathrm{O}_{2}$, have optimal van der Waals interactions with the smallest cage, $5^{12}$ (Figure 1), and preferentially form sII structures that are comprised of $5^{12}$ and $5^{12} 6^{4}$ cages in a $2: 1$ ratio. Slightly larger molecules, with diameters up to $5.9 \AA$, such as methane and $\mathrm{Cl}_{2}$, form sI structures that consist of $5^{12}$ and $5^{12} 6^{2}$ cages in a 1:3 ratio. Larger molecules with diameters up to $6.9 \AA$, such as methylene chloride and tetrahydrofuran, have optimal van der Waals interactions with the larger $5^{12} 6^{4}$ cages and again form sII structures but with only the $5^{12} 6^{4}$ cages occupied. Even larger molecules, with effective diameters up to $9 \AA$, form a type $\mathrm{H}$ structure that contains $5^{12} 6^{8}$ cages. These structures require helper molecules to fill the smaller cages. Iodine, with a van der Waals diameter of $6.7 \AA$, might have

*Corresponding author. E-mail: gkerensk@uci.edu.

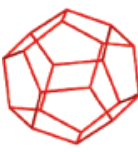

$5^{12}$

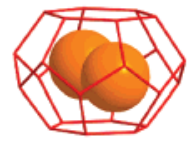

$5^{12} 6^{2}$

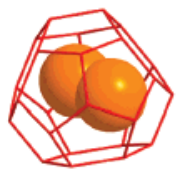

$5^{12} 6^{3}$

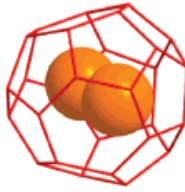

$5^{12} 6^{4}$
Figure 1. Schematic diagrams of four common clathrate hydrate cage types (from left to right): $5^{12}, 5^{12} 6^{2}, 5^{12} 6^{3}$, and $5^{12} 6^{4}$. Cage nomenclature is as follows: 5 and 6 stand for pentagonal and hexagonal faces, respectively, and the superscript gives the number of each face type. For instance, the $5^{12} 6^{4}$ cage has 12 pentagonal faces and 4 hexagonal faces. An oxygen atom is at each vertex, and a hydrogen atom lies along each edge. For perspective, the three larger cages are shown with space-filling bromine molecules inside.

been expected to form sII clathrate hydrate. However, iodine clathrate has not previously been observed, ${ }^{2}$ although both chlorine and bromine hydrates are quite stable. ${ }^{3-7}$ Other large molecules, such as benzene, with $d=7.1 \AA$, can be trapped into sII hydrate with the aid of a helper such as xenon to stabilize the smaller cages of the lattice. ${ }^{8}$ Here, we take the alternate approach of allowing the iodine molecules to substitute for a small fraction of the cage stabilizing molecules.

Since iodine, by itself, does not stabilize the clathrate hydrate lattice but has the appropriate diameter to fit into the $5^{12} 6^{4}$ cages of the sII structure, we chose to make double sII hydrate using the clathrate forming molecules THF, $\mathrm{CH}_{2} \mathrm{Cl}_{2}$, and $\mathrm{CHCl}_{3}$ with iodine as a second guest. The iodine concentration was always less than $1 \%$ of the hydrate forming molecule, and more typically $0.1 \%$. As such, iodine does not significantly affect the stability of the parent hydrate. We also infer that $\mathrm{I}_{2}$ does not induce any structural reorganization of the water cavities and is most likely trapped in large $5^{12} 6^{4}$ cages. Figure 2 shows a slice of a space-filling model for a $5^{12} 6^{4}$ cage occupied by an iodine molecule. 


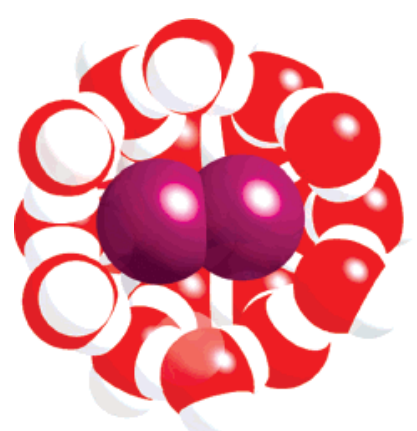

Figure 2. Slice through the space-filling model that illustrates how iodine fits into the $5^{12} 6^{4}$ cage.

In a recent paper, we reported the first UV-vis spectroscopy of another halogen molecule, bromine, trapped in clathrate hydrate cages. ${ }^{7}$ The spectra of bromine inside the clathrate hydrate structures were compared to those of bromine in polar and nonpolar solvents and liquid and frozen water. It was shown that the visible spectra of bromine in the clathrate hydrate cages are more similar to those of gas-phase bromine than to those of bromine in liquid water or amorphous ice. In effect, in the clathrate hydrate cavity, bromine interacts only weakly with the water molecules of the lattice. This is rationalized by the fact that water molecules are fully hydrogen bonded within the clathrate lattice network, so the electron lone pairs on oxygen would not be expected to interact strongly with the halogen.

In the present paper, we report on the UV-vis and resonance Raman spectra of iodine inside sII clathrate hydrates, stabilized by THF, $\mathrm{CH}_{2} \mathrm{Cl}_{2}$, or $\mathrm{CHCl}_{3}$. Changes in the absorption band relative to the gas phase and resonance Raman spectra are used to gain insight into trapping sites and interactions. We tested predictions of molecular mechanics force-field calculations of the water cage-guest association energy. Finally, an interesting aspect of the iodine doped THF clathrate hydrate is the ability to control the ionic equilibrium of the sample. We show that the $\mathrm{I}_{2} / \mathrm{I}_{3}{ }^{-}$equilibrium shifts from the $\mathrm{I}_{3}{ }^{-}$anion in a liquid THF/ water solution to neutral $\mathrm{I}_{2}$ upon crystallization.

\section{Experimental Procedures}

The experimental techniques employed in this study were similar to those described previously for studies of bromine in clathrate hydrate cages. ${ }^{7}$ Two differences are notable: (a) we were not able to prepare clathrate hydrate samples with iodine as the only guest and (b) we have recorded resonance Raman spectra that provide a unique signature of enclathration.

Sample Preparation. Nanopure distilled water (resistivity greater than $16 \mathrm{M} \Omega$ ) was used in all clathrate hydrate experiments. The stated manufactured purity of tetrahydrofuran (THF, $\left.\mathrm{C}_{4} \mathrm{H}_{8} \mathrm{O}\right)$, cyclohexane $\left(\mathrm{C}_{6} \mathrm{H}_{12}\right), n$-hexane $\left(\mathrm{C}_{6} \mathrm{H}_{14}\right)$, carbon tetrachloride $\left(\mathrm{CCl}_{4}\right)$, chloroform $\left(\mathrm{CHCl}_{3}\right)$, and methylene chloride $\left(\mathrm{CH}_{2} \mathrm{Cl}_{2}\right)$ was greater than $99 \%$. No further purification was performed. Samples of iodine doped THF sII clathrate hydrates were obtained both from the liquid phase or by slow formation from the gas phase at temperatures around $0{ }^{\circ} \mathrm{C}$. The latter procedure produces hydrate crystals of optical quality from light pink to light coral depending on the iodine concentration. The crystals can be grown in the optical cuvette directly, but this yields crops of crystals of different shapes and orientations. The resulting light scattering makes this method inappropriate for spectroscopic measurements. Separation of a single crystal is a delicate process requiring a special setup. Therefore, we mainly employed samples grown from the liquid solution to expedite the preparation of samples that could be used for optical studies.
THF clathrate hydrate doped with iodine was prepared from the melt of crystals grown in the gas phase to preserve the THF and iodine mole fraction. The ideal stoichiometry for the sII hydrate is one guest molecule per 17 water molecules. From the optical density of the sample, we estimated that the iodine/ THF ratio was approximately 0.001 , so that the iodine concentration was $0.002 \mathrm{M}$. The liquid solution of iodine in the THF/ water mixture was yellow, which changed to rose upon crystallization. For the THF hydrates, the solution was cooled in the optical cell directly, and a quite uniform polycrystalline sample formed upon a few cycles of temperature around the hydrate melting point, $4.4^{\circ} \mathrm{C} .{ }^{9}$ As shown in Figure 3, the color change reverses upon melting.

Because dichloromethane and trichloromethane are not very soluble in water, a more complicated technique was required to form acceptable samples of iodine doped $\mathrm{CHCl}_{3}$ and $\mathrm{CH}_{2-}$ $\mathrm{Cl}_{2}$ clathrate hydrates. For $\mathrm{CH}_{2} \mathrm{Cl}_{2}$, seed crystals were first formed by flash cooling of a near stoichiometric water- $\mathrm{CH}_{2}$ $\mathrm{Cl}_{2}$ emulsion $\left(\mathrm{CH}_{2} \mathrm{Cl}_{2} \cdot n \mathrm{H}_{2} \mathrm{O}, 16<n<17\right.$, with about $10^{-3}$ $\mathrm{I}_{2} / \mathrm{CH}_{2} \mathrm{Cl}_{2}$ ) at the bottom of a flask. Then, under constant mixing at $0{ }^{\circ} \mathrm{C}$, most of the mixture was converted into an opaque clathrate hydrate ice. Again, the melting point of the sample was the same as that of pure $\mathrm{CH}_{2} \mathrm{Cl}_{2}$ hydrate, $1.7^{\circ} \mathrm{C} .{ }^{9} \mathrm{~A}$ similar procedure was followed for $\mathrm{CHCl}_{3}$. The optical quality of the $\mathrm{CH}_{2} \mathrm{Cl}_{2}$ and $\mathrm{CHCl}_{3}$ hydrate samples never achieved the same degree of quality of those of the THF hydrate.

Gradual freezing of an aqueous iodine solution caused segregation of the iodine from the ice bulk. So, samples of iodine in ice were prepared by vapor deposition of the premixed gases, in the ratio $\mathrm{I}_{2} / \mathrm{H}_{2} \mathrm{O}=1: 500$, on a $0.5 \mathrm{~mm}$ sapphire window held at $120 \mathrm{~K}$, as described previously for bromine. ${ }^{10}$ The vapor deposition technique was expected to yield an amorphous arrangement of the water and iodine molecules because the temperature was low enough to prevent annealing of the sample.

UV-vis and Resonance Raman Spectroscopy. Absorption measurements in the 190-700 $\mathrm{nm}$ spectral range were conducted with a Cary UV-vis spectrometer. Spectra of the $\mathrm{I}_{2}$ doped THF clathrate hydrate were recorded in a temperature range between -20 and $+4{ }^{\circ} \mathrm{C}$. The optical cell consisted of two windows and a $1 \mathrm{~mm}$ Teflon spacer pressed together using Teflon grease as a sealant. To cool the sample, the cell was placed vertically against a thermoelectric (TE) element using thermal grease. The TE element had an $8 \mathrm{~mm}$ diameter hole in the center for the light absorption measurements. The temperature was controlled using a resistance temperature detector (RTD) probe placed on the TE element. A different procedure was chosen for the $\mathrm{I}_{2}$ doped $\mathrm{CHCl}_{3}$ and $\mathrm{CH}_{2} \mathrm{Cl}_{2}$ clathrate hydrates. The pre-made samples were loaded between two windows at $0{ }^{\circ} \mathrm{C}$ and inserted into a quartz Dewar cooled by liquid $\mathrm{N}_{2}$ vapors. The Dewar was then inserted into the spectrometer. The samples never annealed under our experimental conditions, which resulted in a substantial scattering background. Absorption spectra of $\mathrm{I}_{2}$ in solution were recorded using a conventional $10 \mathrm{~mm}$ quartz cuvette at $293 \mathrm{~K}$.

Raman spectra of $\mathrm{I}_{2} / \mathrm{THF}$ hydrate samples were detected at $90^{\circ}$ to the incident light of a $\sim 50 \mathrm{~mW}, 532 \mathrm{~nm}$ excitation laser. A CCD detector (Andor Technology) with a 1/4 m monochromator (Oriel) was used to disperse and detect the spectra. The $250-2000 \mathrm{~cm}^{-1}$ spectral range was calibrated with the bands of iodine in $\mathrm{CCl}_{4}$ and was checked against the known Raman spectra of DMSO, $d$-choroform, and $n$-hexane. The uncertainty in the band positions was $\pm 1 \mathrm{~cm}^{-1}$. A holographic notch filter with an $\sim 180 \mathrm{~cm}^{-1}$ bandwidth was used to filter the scattered laser light. 

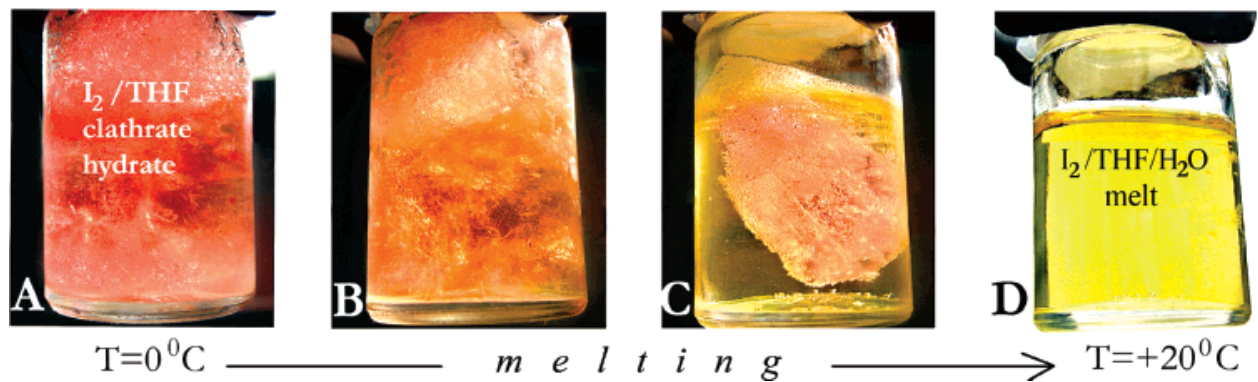

Figure 3. Four images illustrating the color change upon melting for the $\mathrm{I}_{2}$ doped THF clathrate hydrate. (A) Solid-state sample at $0{ }^{\circ} \mathrm{C}$ has a bright rose color. (B and C) Decomposition of the hydrate at $T>4.4{ }^{\circ} \mathrm{C}$; the color changes due to the appearance of a liquid yellow phase. (D) Resulting liquid phase at $20^{\circ} \mathrm{C}$ is yellow.

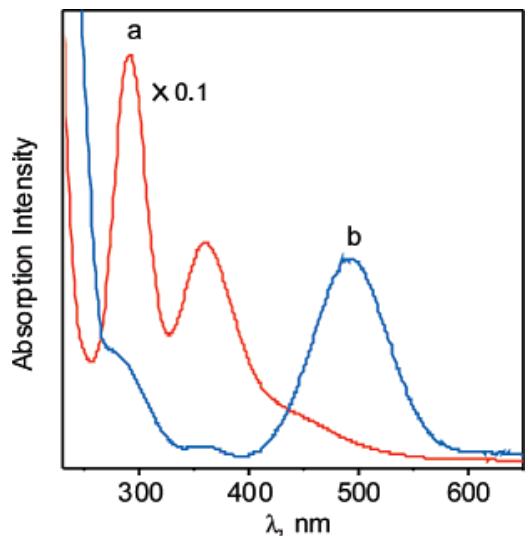

Figure 4. Absorption spectra: (a) $I_{2}$ in water/THF, 17:1, solution and (b) $\mathrm{I}_{2}$ /THF clathrate hydrate $(273 \mathrm{~K})$. Intensity scale of spectrum a is compressed by $90 \%$ to keep its features comparable to those of the $\mathrm{I}_{2} / \mathrm{THF} /$ hydrate spectrum (b).

\section{Results}

Figure 3 shows the remarkable color change upon melting of the $\mathrm{I}_{2}$ doped THF clathrate hydrate. The rose clathrate turned yellow as it decomposed. The process is reversible, and the yellow iodine/THF/water solution turned rose again upon clathrate hydrate formation below $4.4^{\circ} \mathrm{C}$. The main absorption features responsible for the color change are depicted in Figure 4. The spectrum of the liquid iodine/THF/water solution, shown in trace a in Figure 4, is dominated by the absorptions of the triiodide anion, $\mathrm{I}_{3}{ }^{-}$, at 291 and $360 \mathrm{~nm} .{ }^{11,12}$ These ionic bands are quite intense, so the intensity scale of the spectrum (a) was compressed by $90 \%$ to keep its features comparable to those of the $I_{2} / T H F /$ hydrate spectrum, trace $b$ in Figure 4 . As a consequence of hydrate formation, the ionic bands disappeared almost completely, and a band centered at $492 \mathrm{~nm}$ appeared. The latter can be assigned to the valence transitions of iodine. The absorption maximum was blue-shifted by $1440 \mathrm{~cm}^{-1}$ relative to the peak of the valence band in the free molecule. In an aqueous solution of iodine, $\mathrm{I}_{3}{ }^{-}$bands appeared at 287 and $352 \mathrm{~nm}$, respectively, and the molecular $\mathrm{I}_{2}$ absorption peaked at $460 \mathrm{~nm}$. The equilibria involved in triiodide ion formation are

$$
\begin{gathered}
\mathrm{I}_{2}+\mathrm{H}_{2} \mathrm{O} \leftrightarrow \mathrm{HI}+\mathrm{HOI} \\
\mathrm{HI}+\mathrm{H}_{2} \mathrm{O} \leftrightarrow \mathrm{H}_{3} \mathrm{O}^{+}+\mathrm{I}^{-} \\
\mathrm{I}_{2}+\mathrm{I}^{-} \leftrightarrow \mathrm{I}_{3}^{-}
\end{gathered}
$$

Adding THF to a solution of $\mathrm{I}_{2}$ in water shifted the equilibrium further toward ion formation. ${ }^{13}$ For neat THF, a high concentration of iodine leads to ion-catalyzed polymerization. However,

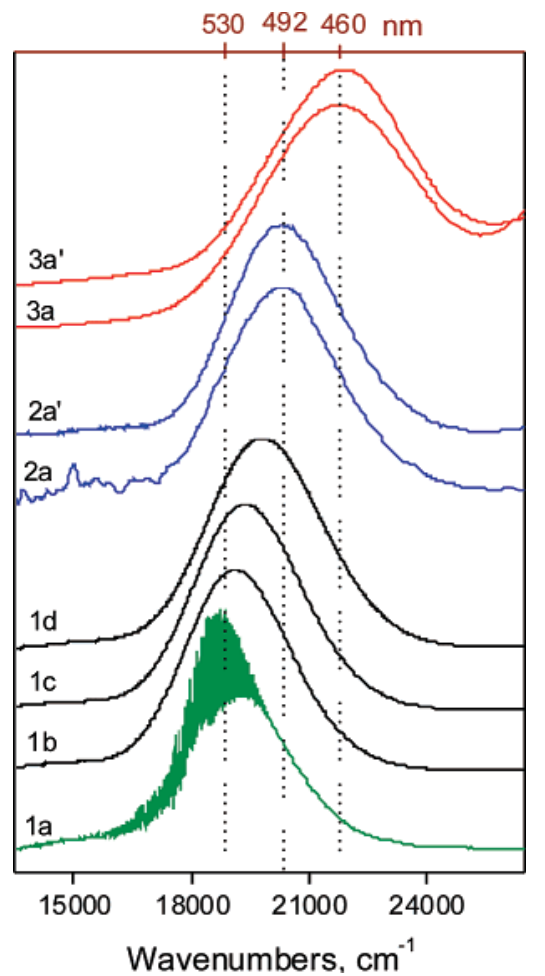

Figure 5. Absorption spectra of $I_{2}$ in (1a) gas phase (data points were taken from the supporting materials of ref 39) (295 K), (1b) $\mathrm{C}_{6} \mathrm{H}_{12}$ (293 K), (1c) $\mathrm{CCl}_{4}(293 \mathrm{~K}),(1 \mathrm{~d}) \mathrm{CH}_{2} \mathrm{Cl}_{2}(293 \mathrm{~K}),(2 \mathrm{a}) \mathrm{I}_{2} / \mathrm{CH}_{2} \mathrm{Cl}_{2}$ clathrate hydrate $(273 \mathrm{~K}),\left(2 \mathrm{a}^{\prime}\right) \mathrm{I}_{2} / \mathrm{THF}$ clathrate hydrate $(273 \mathrm{~K}),(3 \mathrm{a})$ aqueous solution $(293 \mathrm{~K})$, and (3a') amorphous ice (120 K).

in the aqueous solutions at room temperature, the low iodine concentration resulted in negligible permanent chemistry. The freeze-thaw cycle could be repeated several times without changing the intensity of the iodine clathrate hydrate band produced upon formation of the solid. A similar phenomenon occurred for $\mathrm{SO}_{2}$ clathrate hydrate formation. Upon freezing, the bisulfite ions $\left(\mathrm{HSO}_{3}{ }^{-}\right)$in the $\mathrm{SO}_{2}$ aqueous solution disappeared, leaving neutral $\mathrm{SO}_{2}$ molecules inside the clathrate hydrate lattice. ${ }^{14}$

The visible region of the iodine spectrum is defined by transitions from the ground electronic state to the valence excited states, and it is centered at $530 \mathrm{~nm}$ for the gas-phase molecule. The details of the underlying transitions are given in the Discussion. Spectra for these valence transitions of iodine in a variety of environments are shown in Figure 5. Table 1 lists the maxima of the absorption bands, along with bandwidth and shifts relative to iodine in the gas phase for each of the traces in Figure 5. In addition to the visible absorption band, the condensed-phase spectra exhibited strong charge-transfer transitions at wavelengths shorter than $300 \mathrm{~nm}$ (not shown). 
TABLE 1: Parameters of the Valence Absorption Band of $I_{2}$ in Different Media $\left(\mathrm{cm}^{-1}\right)$

\begin{tabular}{lcrc}
\hline & $\omega_{\max }{ }^{a}$ & $\Delta \omega_{\max }{ }^{b}$ & fwhm \\
\hline gas $^{c}$ & 18870 & 0 & 3060 \\
cyclohexane, $\mathrm{C}_{6} \mathrm{H}_{12}$ & 19120 & 250 & 3160 \\
$\mathrm{CCl}_{4}$ & 19360 & 490 & 3270 \\
$\mathrm{CH}_{2} \mathrm{Cl}_{2}$ & 19800 & 930 & 3440 \\
sII hydrate & 20310 & 1440 & 3360 \\
amorphous ice, $T=120 \mathrm{~K}$ & 21870 & 3000 & 4450 \\
aqueous solution, $T=293 \mathrm{~K}$ & 21690 & 2820 & 4500 \\
${ }^{a} \omega_{\text {max }}$ is the position of the absorption maximum, $\pm 50 \mathrm{~cm}^{-1} \cdot{ }^{b} \Delta \omega_{\max }$ \\
is the shift of the absorption maximum from the gas-phase value. \\
${ }^{c}$ fwhm is the full width at half-maximum, $\pm 100 \mathrm{~cm}^{-1} \cdot{ }^{d}$ Spectrum \\
obtained from the appendix data of ref 39.
\end{tabular}

In the nonpolar solvents $\mathrm{C}_{6} \mathrm{H}_{12}$ (Figure 5, 1b) and $\mathrm{CCl}_{4}$ (Figure 5, 1c), the peak of the absorption band was at $520 \mathrm{~nm}$, slightly blue-shifted from the gas phase. Solute-solvent interactions eliminated the discrete vibrational structure of the gasphase spectrum, yielding a continuous absorption band. In polar solvents, the iodine absorption band shifted further to the blue. The effect led to an observable color change. $\mathrm{I}_{2}$ in $\mathrm{CH}_{2} \mathrm{Cl}_{2}$ absorbed at around $500 \mathrm{~nm}$ (Figure 5, 1d) and had a rose color, while $\mathrm{I}_{2}$ in aqueous solution (Figure 5, 3a) and amorphous ice (Figure 5, 3a') absorbed at $460 \mathrm{~nm}$ and had a yellow color. The visible absorption band of $\mathrm{I}_{2}$ in amorphous ice at $120 \mathrm{~K}$ closely resembles that of the aqueous solution, suggesting that the interactions responsible for the strong blue-shift have a similar origin in both water and ice.

When iodine is trapped in the sII clathrate hydrate (Figure 5, $2 \mathrm{a}$ and $\left.2 \mathrm{a}^{\prime}\right)$, the $\mathrm{I}_{2}$ absorption maximum appears at $492 \mathrm{~nm}$, shifted by $1440 \mathrm{~cm}^{-1}$ to the blue of the gas-phase peak. The spectrum was also recorded for $\mathrm{I}_{2}$ doped $\mathrm{CHCl}_{3}$ and $\mathrm{CH}_{2} \mathrm{Cl}_{2}$ sII hydrates, and the absorption maximum was again at 492 $\mathrm{nm}$. The fact that the spectrum of iodine in sII hydrate is independent of the helper molecule indicates that the iodine environment is similar in each structure. Also, since the spectral broadening $\left(300 \mathrm{~cm}^{-1}\right)$ is considerably less than for the aqueous solution or in amorphous ice $\left(1400 \mathrm{~cm}^{-1}\right)$, the local environment of the iodine must be well-defined. We interpret this to indicate that $\mathrm{I}_{2}$ occupies the $5^{12} 6^{4}$ hydrate cage in each structure. Iodine is certainly too large to fit into the smaller $5^{12}$ cages.

Resonance Raman Spectra of Enclathrated Iodine. Figure 6 shows Raman measurements performed on the liquid and frozen THF/water samples with and without iodine. The upper panel shows traces of a liquid THF/water mixture (Figure 6, 1a) and THF/water liquid with iodine (Figure 6, 1b) at $283 \mathrm{~K}$. The two liquid-phase spectra look very similar, except for a shoulder at around $200 \mathrm{~cm}^{-1}$ for the sample containing iodine. Subtracting trace $1 \mathrm{a}$ from $1 \mathrm{~b}$ in Figure 6 yields a distinct peak at $203 \mathrm{~cm}^{-1}$, along with two smaller peaks at about 406 and $625 \mathrm{~cm}^{-1}$. The peak at $406 \mathrm{~cm}^{-1}$ would be the first overtone of the $203 \mathrm{~cm}^{-1}$, which we tentatively assign to the $I_{2}$ stretch of the $\mathrm{I}_{2}-\mathrm{THF}$ complex. The peak at $625 \mathrm{~cm}^{-1}$ cannot be assigned to complexed iodine. It is more likely to be the perturbed ringbend motion of the complexed THF, which in the neat liquid occurs at $658 \mathrm{~cm}^{-1} \cdot 15$

When the solutions, used to obtain spectra 1a and $1 \mathrm{~b}$ in Figure 6, are cooled to below $4.4{ }^{\circ} \mathrm{C}$, they become supercooled with respect to the clathrate hydrate formation. At $-3{ }^{\circ} \mathrm{C}$, the clathrate formation starts, and we observe sample heating $(\Delta T$ $=\sim 1-1.5^{\circ}$ ) before the temperature stabilizes at the set value. The spectra then change to those shown in Figure 6, traces $2 \mathrm{a}$ and $2 \mathrm{~b}$. Now, the differences between the two spectra are easily observed. Trace 2a in Figure 6 is the THF clathrate hydrate
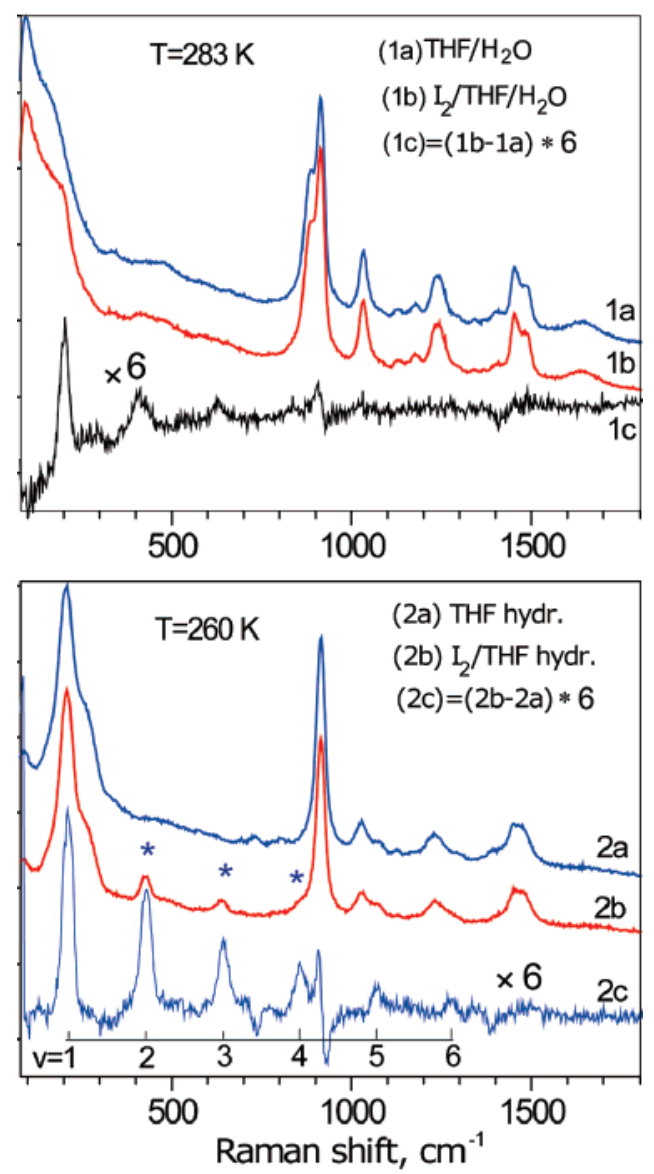

Figure 6. Raman spectra of $\mathrm{THF} / \mathrm{H}_{2} \mathrm{O}$ and $\mathrm{I}_{2} / \mathrm{THF} / \mathrm{H}_{2} \mathrm{O}$ samples at 283 and $260 \mathrm{~K}$, respectively. Molar ratio of THF/water is 1:17. Samples were obtained from melt of the gas-phase grown hydrate crystals to preserve the mol fraction. Iodine concentration was estimated to be 0.002 M. (1a) Liquid THF aqueous solution, (1b) liquid THF aqueous solution with iodine, (1c) difference between $1 \mathrm{~b}$ and $1 \mathrm{a}$ multiplied by a factor of 6, (2a) THF clathrate hydrate, (2b) $\mathrm{I}_{2}$ doped THF clathrate hydrate, and (2c) difference between $2 \mathrm{~b}$ and $2 \mathrm{a}$ multiplied by a factor of 6 .

without iodine, while trace $2 b$ in Figure 6 is the THF clathrate hydrate with iodine. Since the samples are not fully annealed, there may be some remaining ice. Two peaks of the iodine progression at 427 and $641 \mathrm{~cm}^{-1}$ are clearly visible in trace $2 \mathrm{~b}$ in Figure 6, but the iodine Raman signal is still weak since the $\mathrm{I}_{2}$ concentration in the sample is only $0.1 \%$ of the THF concentration. The detection of the Raman signal is possible only due to the resonance enhancement at $532 \mathrm{~nm}$. Subtracting trace $2 \mathrm{a}$ from trace $2 \mathrm{~b}$ in Figure 6 yields a clear progression in the iodine stretch up to the fifth overtone, as indicated in trace $2 \mathrm{c}$ in Figure 6. Note the monotonic decrease in overtone line intensities. The linewidths are limited by the experimental resolution. A Birge-Sponer fit to the energy difference between two adjacent lines, $\omega_{v+1, v}$, yields $\omega_{\mathrm{e}}=214 \pm 1 \mathrm{~cm}^{-1}$, which, within experimental error, is the same as the gas-phase value $\left(214.5 \mathrm{~cm}^{-1}\right)$. For comparison, $\omega_{\mathrm{e}}=212.5$ and $209.6 \mathrm{~cm}^{-1}$ in $\mathrm{CCl}_{4}$ and $\mathrm{CHCl}_{3}$, respectivley. ${ }^{16,17}$ Unfortunately, we were not able to obtain a reliable anharmonicity constant, $\omega_{\mathrm{e}} x_{\mathrm{e}}$, but it appears that $\omega_{\mathrm{e}} x_{\mathrm{e}}$ may be even less than in the gas phase, 0.6 $\mathrm{cm}^{-1}$. That the molecule is only slightly perturbed by the trap site is reinforced by the widths of the lines in the resonance Raman progression. From the resolution-limited linewidths, we may establish that vibrational dephasing times are longer than 1 ps up to $v=6$. Given the light mass of $\mathrm{H}_{2} \mathrm{O}$ and its fast internal degrees of freedom, the long dephasing time indicates 
that $I_{2}$ is mechanically decoupled from the water lattice-the guest is trapped in a cage to which it is poorly coupled. In contrast with the enclathrated iodine, resonance Raman spectra of iodine in aqueous solution could not be obtained for a saturated sample, $\sim 0.001 \mathrm{M}$. This failure may be ascribed to a combination of much faster dephasing (electronic and/or vibrational) and with insufficient resonance enhancement in water as compared to the clathrate hydrate.

The assignments for some of the THF clathrate hydrate peaks are discussed by Manakov et al. ${ }^{18}$ and Tulk et al. ${ }^{19}$ The strongest peak at $914 \pm 1 \mathrm{~cm}^{-1}$ in the THF aqueous solution belongs to the THF breathing $\mathrm{C}-\mathrm{C}-\mathrm{C}-\mathrm{C}$ mode. This band acquires a shoulder at $887 \mathrm{~cm}^{-1}$ due to the hydrogen bonded THF-water complex. Notice that the shoulder disappears upon clathrate hydrate formation and that the main THF peak shifts by $1 \mathrm{~cm}^{-1}$ to higher frequency. In the low-frequency region, the broad band belongs to the intermolecular $\mathrm{O}-\mathrm{O}$ stretching vibration of water. The band changes its shape and position upon freezing, as was previously observed. ${ }^{20}$

One final observation is worth mentioning. When the rose iodine doped THF clathrate samples were cooled to liquid nitrogen temperatures, they changed to pale yellow. The color was restored upon warming. We cannot explain this phenomenon at present.

\section{Discussion}

UV-vis Spectroscopy. As illustrated in Figure 5, the visible absorption band of iodine is a sensitive probe of the local environment. Going from the gas phase to the clathrate hydrate phase, the iodine valence band absorption peak blue-shifts by $1440 \mathrm{~cm}^{-1}$, while from the gas phase to the aqueous solution, the blue-shift is $3000 \mathrm{~cm}^{-1}$. Table 1 shows that usually the larger the shift, the broader the spectrum. A significant exception to this trend is the width of the iodine spectrum in clathrate hydrate, which is less than that of the iodine spectrum in $\mathrm{CH}_{2} \mathrm{Cl}_{2}$, even though the blue-shift is greater for clathrate. In water and ice, the spectral shift is accompanied by the dramatic broadening, $\sim 50 \%$, whereas in clathrate hydrates, the width of the band increases by less than $10 \%$. As such, the essential features of the spectra that we will discuss are the fact that the enclathrated iodine spectra are significantly blue-shifted, although not nearly so much as in aqueous solution, while the width of the enclathrated iodine spectrum is only slightly greater than in the nonpolar solvents. We also note that for bromine in a clathrate hydrate $5^{12} 6^{4}$ cage, the same one studied here, the corresponding shift was only $360 \mathrm{~cm}^{-1}$, one-fourth of that observed for iodine.

Analysis of the spectral shift and broadening is complicated by the fact that three separate valence transitions underlie the band: $\mathrm{C}, \mathrm{B}$, and $\mathrm{A} \leftarrow \mathrm{X}$. Each of these transitions formally involves excitation of an electron from the $\pi^{*}$ orbital, perpendicular to the iodine bond to the $\sigma^{*}$ orbital on the end of the molecule. They differ in the details of the spin-orbit coupling. The $\mathrm{B}^{3} \Pi_{0 \mathrm{u}} \leftarrow \mathrm{X}^{1} \Sigma^{+}{ }_{\mathrm{g}}$ transition, which accounts for $70 \%$ of the intensity in the gas-phase spectrum, ${ }^{21}$ exhibits considerable vibrational substructure. The $\mathrm{C}^{1} \Pi_{1 \mathrm{u}} \leftarrow \mathrm{X}^{1} \Sigma^{+}{ }_{\mathrm{g}}$ transition accounts for nearly $30 \%$ of the gas-phase intensity and is featureless since it is a bound-free transition. A weak $\mathrm{A}^{1} \Pi_{1 \mathrm{u}} \leftarrow \mathrm{X}^{1} \Sigma^{+}{ }_{\mathrm{g}}$ transition contributes to the red tail of the band. The condensed-phase spectra are too smooth to allow for a direct decomposition in terms of underlying contributions from the various valence transitions. Nevertheless, it is important to note that multiple electronic surfaces are involved and that a change in the shift and width of any given spectrum is a combination of shifting the energy of the states and changing of the relative intensities

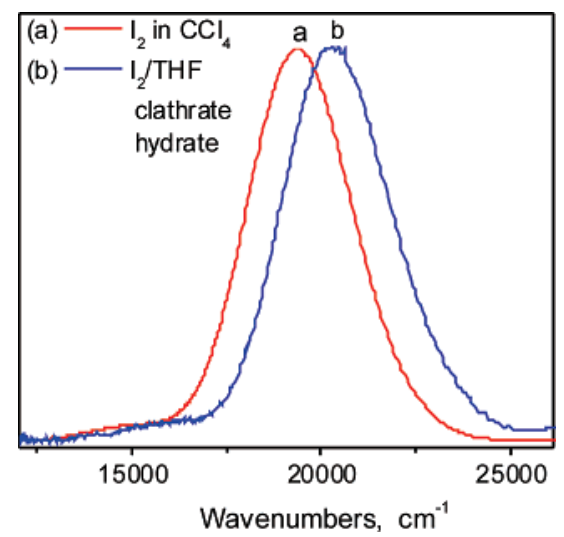

Figure 7. Absorption spectra of $I_{2}$ in (a) $\mathrm{CCL}_{4}(295 \mathrm{~K})$ and (b) $\mathrm{I}_{2} /$ THF clathrate hydrate $(273 \mathrm{~K})$.

of the underlying transitions. Here, we will consider the principal differences between iodine in sII clathrate hydrate cages and iodine dissolved in water or ice and discuss the various contributions to the observed changes in the spectrum.

Although the changes in the spectra in aqueous solution and amorphous ice are more dramatic, they may be understood, at least qualitatively, in terms of nearest neighbor interactions. In analogy with $\mathrm{H}_{2} \mathrm{O}-\mathrm{Cl}_{2}{ }^{22}$ and $\mathrm{H}_{2} \mathrm{O}-\mathrm{Br}_{2}$ dimers, ${ }^{23}$ the ground state is expected to be stabilized by a strong $\mathrm{O}-\mathrm{I}$ bond between the nearest neighbor water molecule and the iodine molecule. The $\mathrm{H}_{2} \mathrm{O}-\mathrm{Cl}_{2}$ and $\mathrm{H}_{2} \mathrm{O}-\mathrm{Br}_{2}$ well depths are about 2 and $3 \mathrm{kcal} /$ mol, respectively; ${ }^{24}$ if $\mathrm{H}_{2} \mathrm{O}-\mathrm{I}_{2}$ follows this trend, the $\mathrm{O}-\mathrm{I}$ bond should be even stronger. The short $\mathrm{O}-\mathrm{I}$ bond length in the ground state leads to a repulsive interaction in the excited state, for which the newly filled $\sigma^{*}$ orbital will repel the lone electron pairs on the water molecule. The dramatic broadening of the band in water can be understood by the fact that the formation of the $\mathrm{O}-\mathrm{I}$ bond in the ground state results in significant polarization of the iodine moiety. Thus, the transition dipole is no longer limited to the $\mathrm{I}_{2}$ internuclear coordinate: the $\mathrm{I}-\mathrm{O}$ coordinate also acquires a transition dipole. If we assume the separability of coordinates, then the overall spectrum is obtained as the convolution of the two vertical transitions, with the result that the overall spectral width is given as $\sigma=\left[\sigma_{1}^{2}+\sigma_{2}^{2}\right]^{1 / 2}$, where $\sigma_{1}$ and $\sigma_{2}$ are spectral widths along the $\mathrm{I}-\mathrm{I}$ and $\mathrm{I}-\mathrm{O}$ coordinates. The observed $50 \%$ increase in bandwidth implies that the contribution from each coordinate is similar. Since the spectral width is determined by the gradient of the excited-state potential at the ground-state geometry, $\sigma \propto \nabla V\left(r=r_{\mathrm{e}}\right)$, the reproduction of the shift and broadening of the transition yields information about the solute-solvent coordinate. Our prior treatment of aqueous $\mathrm{Br}_{2}$ in terms of a single $\mathrm{H}_{2} \mathrm{O}-\mathrm{Br}_{2}$ interaction $^{7,10}$ is applicable to the present discussion. Also, preliminary ab initio results for $\mathrm{H}_{2} \mathrm{O}-\mathrm{Cl}_{2}$ and $\mathrm{H}_{2} \mathrm{O}-\mathrm{Br}_{2}$ indicate that a substantial fraction of the aqueous solvent shift for these species can be attributed to a single nearest neighbor interaction. ${ }^{25}$

The important signature of enclathrated iodine is that the shift in the spectrum is significant, half that of the aqueous solution, while the width of the spectrum remains quite narrow, similar to that in a nonpolar solvent. Figure 7 shows a more detailed comparison of the enclathrated iodine spectrum with that in $\mathrm{CCl}_{4}$ solution. These spectra show that the band shapes are almost identical in two cases. We consider two limiting cases to explain these phenomena. First, to the extent that each of the three underlying transitions is uniformly blue-shifted, the most important contribution to the blue-shift is ground-state stabilization plus excited-state repulsion of the cage walls. If the 
electronic states were simply shifted in energy, there would be no change in the width of the spectrum. The second limiting case is that in the clathrate hydrate, the intensity of the $\mathrm{C} \leftarrow \mathrm{X}$ transition becomes greater than the intensity of the $\mathrm{B} \leftarrow \mathrm{X}$ transition: the opposite situation of the gas phase. This could account for much of the blue-shift, and the spectrum would not be broadened significantly. Such an intensity shift could result from the fact that the excited states might be coupled to chargetransfer and ion-pair states, whose energy will be significantly lowered in the clathrate as compared to the gas phase. It may well be that both of these limiting mechanisms contribute to the observed result. In the following paragraphs, we discuss each of them in slightly more detail.

If state energy shifts are responsible for the observed trends, it is important to note that the observed clathrate blue-shift is significantly larger than for polar $\mathrm{CH}_{2} \mathrm{Cl}_{2}$, yet only half that of liquid water. As discussed earlier for the case of bromine, ${ }^{7}$ it is not surprising that halogen molecules interact more strongly with liquid water molecules than with water molecules in the clathrate cage walls. In the clathrate structure, all water molecules are fully hydrogen bonded, so the lone electron pairs on oxygen atoms are not available for interaction with the guest molecule. Similarly, $\mathrm{H}$ atoms do not point into the cage and therefore are unavailable for direct bonding with the guest. To a first approximation, the electrostatic field near the cage center, which is a vector sum of electrostatic fields of water molecules constituting the cage, can be neglected. ${ }^{26}$ It then seems reasonable to assume that the interaction between hydrate cage and iodine is mainly due to dispersion forces. Roughly, the dispersion forces for water molecules will be similar to those of argon, and thus, the clathrate cage environment might be similar to that of an argon matrix (or other rare gas matrices), for which iodine spectra have been extensively analyzed. ${ }^{27-29}$ However, in rare gas matrices, the shift of the iodine absorption band does not exceed $200 \mathrm{~cm}^{-1}$. Thus, as simple dispersion interactions alone are unlikely to account for the observed clathrate spectra, stronger perturbations are needed. One possibility is that the iodine interacts more strongly than expected with the oxygen atoms. Recent calculations by Schofield and Jordan ${ }^{30}$ of the $\mathrm{Cl}_{2}$ $\left(\mathrm{H}_{2} \mathrm{O}\right)_{20}$ cluster, which mimics $\mathrm{Cl}_{2}$ in the $5^{12}$ cage, indicate that the hydrogen bonding in a $5^{12}$ cage does not eliminate the ability of the oxygen atoms to donate electron density to the $\sigma^{*}$ orbital on a chlorine molecule. The chlorine prefers to align on an axis between two oxygen atoms. A similar interaction may lead to significant ground-state stabilization for iodine in the $5^{12} 6^{4}$ cage. Another possibility is that the electrostatic interactions may be relatively strong for the iodine atoms near the cage walls.

Next, we consider the role of changing the relative intensities of the $\mathrm{C} \leftarrow \mathrm{X}$ and $\mathrm{B} \leftarrow \mathrm{X}$ transitions. In an analysis of the $\mathrm{I}_{2}$ absorption spectra in $\mathrm{CCl}_{4}$ and $n$-heptane, ${ }^{21,31}$ it was proposed that, in $n$-heptane, the intensity of the $\mathrm{C} \leftarrow \mathrm{X}$ component is doubled relative to the gas phase. This results in an effective spectral shift of the absorption spectrum. The observed peak position of iodine in the clathrate hydrate corresponds to the maximum of the gaseous $\mathrm{I}_{2} \mathrm{C} \leftarrow \mathrm{X}$ transition, so it is tempting to attribute the majority of the observed spectral shifts to a dramatic enhancement of the $\mathrm{C}$ state over the $\mathrm{B}$ state. However, as shown in Figure 7, which compares the spectra for iodine in clathrate hydrate to that in $\mathrm{CCl}_{4}$, the weak $\mathrm{A} \leftarrow \mathrm{X}$ band on the red side of the spectrum is shifted in the clathrate environment in accord with the main feature. Therefore, it would be difficult to imagine why the position of the $\mathrm{C} \leftarrow \mathrm{X}$ band practically does not change, while the $\mathrm{A} \leftarrow \mathrm{X}$ band shifts by about $1000 \mathrm{~cm}^{-1}$. Figure 7 also demonstrates how similar the shapes of the spectra are in the two media. Any substantial enhancement of the $\mathrm{C} \leftarrow$ $\mathrm{X}$ transition will tend to change the observed band shape.

We are left with the interesting phenomenon that the signature of enclathration for a halogen molecule is a blue-shift in the valence absorption spectrum with little change in the width of the spectrum. Also, the Raman spectrum of the halogen indicates little perturbation of the bond, as is discussed next. Most likely, the final explanation for these interesting phenomena will involve a combination of the effects we have discussed, although we expect that the relative solvation of the ground and excited states is the single largest contribution to the observed spectral shifts. We will pursue models to shed further light on this issue.

Interpretation of the Raman Measurements. The observation of a long resonance Raman progression for iodine inside the clathrate hydrate structure is an indication that the molecule is trapped in a well-defined environment rather than in crystal defects. The monotonic decay of intensities of the progression is similar to that observed in rare gas matrices, although in matrices, longer progressions are observed due to the greater signal/noise ratios. Moreover, the progression indicates that the transition dipole is limited to the molecular coordinate: the excited-state packet first evolves along the molecular coordinate, then couples to the lattice leading to electronic dephasing. Formation of the resonant Raman progression takes place on time scales less than the electronic dephasing time. The decay rate of the iodine progression in clathrate hydrate (i.e., the integral intensity dependence of overtones on the vibrational quantum number) suggests an electronic dephasing time of $\sim 80$ $\mathrm{fs}$, as established in the analysis of the resonance Raman spectra of iodine in rare gas matrices. ${ }^{32}$ For inert solvents such as $\mathrm{CCl}_{4}$ or $n$-hexane, the resonance Raman progression can be obtained up to the 15th - to 20th overtone with a gradual, monotonic intensity decrease. ${ }^{16}$ In contrast, in polar solvents such as $p$-dioxane, only a short resonance Raman progression, up to $v$ $=3$, is observed, and the intensity of the first overtone is no more than $10 \%$ of that of the fundamental transition. ${ }^{17}$ Iodine interacts strongly with $p$-dioxane by making an $\mathrm{I}-\mathrm{O}$ bond with the nearest solvent molecule. Upon $\mathrm{I}_{2}$ electronic excitation, vibrational modes of the solvent are directly excited, resulting in a very fast electronic dephasing of the iodine excited states and resulting in the dramatic shortening of the resonant Raman progression. The extreme case occurs in water, where the direct coupling between water and $\mathrm{I}_{2}$ completely eliminates the resonance Raman spectrum. For the enclathrated iodine, where the molecule does not form bonds with water and thus the lattice modes are not involved in the iodine electronic excitation, a progression up to $v=6$ is observed, and the intensity falls off gradually with the increasing overtone number. (The number of observed overtones in the progression is determined by the signal/noise ratio in the experiment.) In this respect again, the dynamics of iodine in the sII clathrate lattice is more similar to inert solvents or rare gas matrices than it is to aqueous solution. The ground electronic state of iodine is only slightly perturbed in clathrate hydrate.

Force-Field Simulations of the Halogen-Clathrate Hydrate Cage Association Energy. Although there are many studies of which molecules fit into each of the hydrate clathrate cage types, including a considerable amount of thermodynamic information and an increasing number of spectroscopic studies, the details of how any particular molecule contributes to clathrate hydrate stability have yet to be worked out. In a 1992 analysis of hydrate dissociation enthalpy and guest size, Sloan and Fleyfel concluded, "To a first approximation, the enthalpy of dissociation is independent of the type and concentration of 
the guest gas, within a broad size constraint." 33 Part of the reason for this is that the largest contribution to the enthalpy comes from the water lattice. Hydrate stability represents a delicate balance between enthalpy and entropy effects, and the net enthalpy is due to a delicate balance between positive and negative contributions to the enthalpy for hydrate formation. More recently, White et al. ${ }^{34}$ analyzed the temperature dependence of the enthalpy, entropy, and free energy of formation for THF clathrate hydrate and ethylene oxide clathrate hydrate. They find that enthalpy favors clathrate formation at low temperatures and especially above the melting point of the guest molecule. This suggests that the guest-host association energy for hydrate clathrate should not only be negative, it should be more negative than the enthalpy of condensation for the liquid phase of the guest species.

The halogen hydrates present an interesting special case for observing trends in the dependence of hydrate stability on molecular size and shape. Chlorine fits tightly into the small $5^{12}$ cage and easily into the larger $5^{12} 6^{2}$ cages. ${ }^{5}$ As a result, chlorine hydrate forms a very stable sI structure that consists of a 1:3 ratio of these two cage sizes. Bromine is too large to fit into the small $5^{12}$ cage but still fits well into the $5^{12} 6^{2}$ cages. However, even though the $5^{12} 6^{2}$ cages dominate the sI structure, bromine does not make an sI clathrate. Instead, it makes a more complicated clathrate with a 5:8:2 ratio of $5^{12}, 5^{12} 6^{2}$, and $5^{12} 6^{3}$ cages. ${ }^{6}$ From this, we infer that bromine is especially stable in the $5^{12} 6^{3}$ cages. Iodine, on the other hand, cannot induce clathrate formation by itself, although iodine fits well into the $5^{12} 6^{4}$ cages.

It might seem that modern ab initio methods might soon start to shed light on the formation thermodynamics of a variety of clathrate hydrates, at least at low temperature. ${ }^{35}$ However, to accurately calculate the van der Waals interactions that are important for these systems, both large basis sets and high order electron correlation are necessary. Such calculations are clearly not feasible, for instance, for iodine in a $5^{12} 6^{4}$ cage, even if the rest of the lattice is ignored. Similarly, neither density functional nor semiempirical molecular orbital methods are well-suited for calculating van der Waals forces. Instead, here we present the results of a simple model using the Merck Molecular Force Field $(\mathrm{MMFF})^{36}$ that was developed to simulate intermolecular interactions for drug design calculations. This force field, based on Lennard-Jones interactions exclusively, is expected to yield approximately correct results for van der Waals diameters and well depths. The calculations described next were performed with the Spartan molecular mechanics package. ${ }^{36,37}$

Each of the four cage types, $5^{12}, 5^{12} 6^{2}, 5^{12} 6^{3}$, and $5^{12} 6^{4}$, was constructed by taking the oxygen coordinates from X-ray diffraction data. 8,38 The hydrogen atom positions were then assigned to achieve the proper number of hydrogen bonds while maintaining a small overall dipole moment for the structure and spreading the dangling $\mathrm{OH}$ bonds uniformly around the cage. The cage energy was then optimized with fixed oxygen atom positions. While the cage energy depends on the specific orientations of the dangling $\mathrm{O}-\mathrm{H}$ bonds, we find that the guesthost association energy is less sensitive to the assumed dangling bond distribution. After constructing each cage type, the positions for all of the water molecules were fixed, a halogen molecule was added to the cage, and its orientation was optimized to minimize the total energy. In most cases, the geometry was optimized with the halogen molecule located near the center of the cages. For large cages and small halogens, especially for $\mathrm{F}_{2}$ and $\mathrm{Cl}_{2}$ in the $5^{12} 6^{4}$ cage, the halogen moves closer to one of the cage walls to achieve the most favorable
TABLE 2: Calculated Association Energy for Cage Filling $(\mathrm{kJ} / \mathrm{mol})$

\begin{tabular}{cllll}
\hline halogen/cage type & $5^{12}$ & $5^{12} 6^{2}$ & $5^{12} 6^{3}$ & $5^{12} 6^{4}$ \\
\hline $\mathrm{F}_{2}$ & -8 & -7 & -5 & -5 \\
$\mathrm{Cl}_{2}$ & 22 & -17 & -20 & -17 \\
$\mathrm{Br}_{2}$ & 91 & -6 & -24 & -21 \\
$\mathrm{I}_{2}$ & 316 & 60 & -17 & -18
\end{tabular}

van der Waals interaction. The guest-host association energy was then taken to be equal to the energy of the filled cage minus the sum of the energies for the empty cage and the free halogen molecule. Values obtained are listed in Table 2. Further details regarding the model and tests performed to ensure that the relative energy differences reported in Table 2 are not strongly dependent on the assumptions that were made will be reported in a future publication. We also note that the association energy will be very sensitive to the built-in parameters for the van der Waals interaction, especially the equilibrium intermolecular separation of the model potential.

The MMFF model yields a significant, positive (destabilizing) association energy for chlorine in the $5^{12}$ cage and a negative (stabilizing) energy for the $5^{12} 6^{2}$ cage. This is roughly consistent with the conclusions of Pauling and $\mathrm{Marsh}^{3}$ and Cady ${ }^{4,5}$ that only the large cages of sI chlorine hydrate are significantly occupied. Although chlorine is slightly more stable in the $5^{12} 6^{3}$ cage than in the $5^{12} 6^{2}$ cage, the difference is not enough to induce chlorine to form a bromine-type hydrate structure. In contrast, bromine strongly prefers the $5^{12} 6^{3}$ cage to the $5^{12} 6^{2}$ cage, as expected. Iodine has about the same association energy for the $5^{12} 6^{3}$ and $5^{12} 6^{4}$ cages, but in each case, the association energy is less than that for bromine. The repulsive interaction of iodine with the $5^{12} 6^{2}$ cage keeps it from forming a bromine hydrate structure, and the attraction to $5^{12} 6^{4}$ is too weak for forming an sII structure on its own but does not impede iodine molecules from occasionally substituting for other molecules in the sII structure. The association energy for iodine in the $5^{12} 6^{4}$ cage is less than that of bromine, indicating a stronger repulsion interaction with the cage wall.

The fact that iodine readily substitutes for THF in an sII clathrate hydrate, but is less stable than bromine in the $5^{12} 6^{4}$ cage, is consistent with the inability of iodine to form an sII clathrate. Since the $5^{12} 6^{4}$ cage is nearly spherical, the van der Waals repulsion between a prolate halogen molecule and the spherical cage is limited to a few water molecules on the end of the halogen. This is illustrated in Figure 2. This picture helps to visualize that in the $5^{12} 6^{4}$ cages, most of the water molecules only weakly interact with the iodine even if the fit is rather tight on the ends. We also note that the formation of iodine hydrate is also impeded by the fact that under the formation conditions iodine is a solid, and the heat of fusion, $7.8 \mathrm{~kJ} / \mathrm{mol}$, works against clathrate formation.

Color Change of Aqueous THF Solution upon Freezing. Finally, we emphasize the remarkable color change that occurs when the iodine doped THF clathrate is melted. This reversible color change is a consequence of the $\mathrm{I}_{3}{ }^{-} / \mathrm{I}_{2}$ equilibrium shift upon clathrate hydrate formation. Triiodide anions are not stable in the clathrate cages, while neutral $\mathrm{I}_{2}$ molecules in low concentrations appear not to affect the stability of the hydrate. Consequently, the solution is substantially deionized upon $\mathrm{I}_{2} /$ THF hydrate formation. Simple freezing of an aqueous solution also tends to exclude triiodide anions, but neutral $\mathrm{I}_{2}$ molecules are excluded as well, leaving regular ice with precipitated iodine clusters in imperfections. In the case of the iodine doped THF clathrate, however, there is no separation of the iodine from the water when the solid clathrate hydrate is formed. Instead, 
the effect of enclathration forces the equilibrium to shift away from triiodide toward $\mathrm{I}_{2}$, which is then trapped in the cages.

\section{Conclusion}

We have presented valence absorption spectra and resonant Raman data for iodine in clathrate hydrates and water. The data strongly suggest that iodine molecules can substitute in about $0.1 \%$ of the $5^{12} 6^{4}$ cages of sII clathrates. The resonance Raman spectra in the clathrate hydrates show a progression with intensity decay that is similar to that of the molecule in rare gas solids: the molecular ground state is slightly perturbed, the vibrational dephasing times of $>1 \mathrm{ps}$ indicate that the molecule is poorly coupled to the host cage, and the resonance Raman intensities and dephasing rates indicate that the transition dipole of the molecule is localized on the molecular coordinate. There is an apparent blue-shift of $1440 \mathrm{~cm}^{-1}$ in the absorption band, which is primarily ascribed to the differential solvation, although changes in relative intensity between the underlying transitions may also contribute. In contrast, in water and in ice, the valence bands are broadened by $50 \%$, undergo a blue-shift of $3000 \mathrm{~cm}^{-1}$, and no resonance Raman spectra could be obtained. The observations for water and ice are attributed to strong binding between the $I_{2}$ and a water molecule through the lone pair of electrons of the oxygen atom. The strong polarization of the molecular halogen by this interaction results in the transition dipole being shared between the internal molecular coordinate and the intramolecular halogen-water coordinate, leading to direct excitation of the solute-solvent degree of freedom. The interaction along this coordinate changes from bonding in the ground state to repulsive in the excited electronic states, resulting in a dramatic blue-shift, broadening of the valence transition, and very fast dephasing that precludes the observation of resonance Raman scattering. The UV-vis and resonance Raman spectra serve as insightful tools for characterizing the local environment of the guest molecule--the signature of enclathration is unmistakable. Finally, we have also shown that clathrate formation strongly shifts the phase equilibrium away from triiodide anions toward neutral $\mathrm{I}_{2}$ molecules.

Further work will be necessary to provide a complete analysis of the observed spectroscopy. Although the preliminary force field study presented here is roughly consistent with the relative stability of halogen molecules in the various cages, more sophisticated modeling would be very valuable. Work is in progress to provide similar spectra for chlorine in hydrate cages and also to extend the Raman measurements to other systems. Finally, work is in progress to increase the fractional doping by iodine to allow for an X-ray study of the specific arrangement of water molecules around the iodine moiety.

Acknowledgment. This work was supported by the U.S. National Science Foundation Grant CHE-0404743. We acknowledge discussions with Drs. Ramon Henrnandez and Nadine Halberstadt regarding their calculation of the electronic spectrum of the $\mathrm{H}_{2} \mathrm{O}-\mathrm{Br}_{2}$ dimer and Daniel Schofield and Kenneth Jordan for preliminary communication of their ab initio results for chlorine in $\left(\mathrm{H}_{2} \mathrm{O}\right)_{20}$ and $\left(\mathrm{H}_{2} \mathrm{O}\right)_{24}$ cages.

\section{References and Notes}

(1) Ripmeester, J. A. Ann. N.Y. Acad. Sci. 2000, 912, 1.

(2) Although a mixed $\mathrm{I}_{2} / \mathrm{H}_{2} \mathrm{~S}$ type II clathrate hydrate was referred to in Crystallography in Modern Chemistry; Mak, T. C. W.; Zhou, G.-d.; Wiley-Interscience: New York; Table 7.1-3, p 1178, we have not been able to find any confirmation or elaboration of this result in the primary literature.

(3) Pauling, L.; Marsh, R. E. Proc. Natl. Acad. Sci. U.S.A. 1952, 38, 112.

(4) Cady, G. H. J. Phys. Chem. 1981, 85, 3225

(5) Cady, G. H. J. Phys. Chem. 1983, 87, 4437

(6) Udachin, K. A.; Enright, G. D.; Ratcliffe, C. I.; Ripmeester, J. A. J. Am. Chem. Soc. 1997, 119, 11481 .

(7) Kerenskaya, G.; Goldschleger, I. U.; Apkarian, V. A.; Janda, K. C. J. Phys. Chem. A 2006, 110, 13792 .

(8) Udachin, K. A.; Ratcliffe, C. I.; Ripmeester, J. A. J. Supramol. Chem. 2003, 2, 405.

(9) Davidson, D. W. Water: A Comprehensive Treaties; Plenum: New York, 1973; Vol. 2, pp 115-234.

(10) Goldschleger, I. U.; Senekerimyan, V.; Krage, M. S.; Seferyan, H.; Janda, K. C.; Apkarian, V. A. J. Chem. Phys. 2006, 124, 204507.

(11) Kiefer, W.; Bernstein, H. J. Chem. Phys. Lett. 1972, 16, 5.

(12) Vala, J.; Kosloff, R.; Harvey, J. N. J. Chem. Phys. 2001, 114, 7413

(13) Cataldo, F. Eur. Polym. J. 1996, 32, 1297.

(14) Zhang, Z.; Ewing, G. E. J. Phys. Chem. A 2004, 108, 1681.

(15) Cadioli, B.; Gallinella, E.; Coulombeau, C.; Jobic, H.; Berthier, G. J. Phys. Chem. 1993, 97, 7844 .

(16) Kiefer, W.; Bernstein, H. J. J. Raman Spectrosc. 1973, 1, 417.

(17) Kiefer, W. Appl. Spectrosc. 1974, 28, 115.

(18) Manakov, A. Y.; Goryainov, S. V.; Kurnosov, A. V.; Likhacheva,

A. Y.; Dyadin, Y. A.; Larionov, E. G. J. Phys. Chem. B 2003, 107, 7861. (19) Tulk, C. A.; Klug, D. D.; Ripmeester, J. A. J. Phys. Chem. A 1998, 102,8734

(20) Schicks, J. M.; Erzinger, J.; Ziemann, M. A. Spectrochim. Acta Part A 2005, 61, 2399.

(21) Gray, R. I.; Luckett, K. M.; Tellinghuisen, J. J. Phys. Chem. A 2001, 105, 11183

(22) Davey, J. B.; Legon, A. C.; Thumwood, J. M. A. J. Chem. Phys 2001, 114, 6190 .

(23) Legon, A. C.; Thumwood, J. M. A.; Waclawik, E. R. Chem.-Eur. J. 2002, 8,940.

(24) Alkorta, I.; Rozas, I.; Elguero, J. J. Phys. Chem. A 1998, 102, 9278.

(25) R. Hernández-Lamoneda and N. Halberstadt, personal communication.

(26) Davidson, D. W. Can. J. Chem. 1971, 49, 1224.

(27) Macler, M.; Nicolai, J. P.; Heaven, M. C. J. Chem. Phys. 1989, 91,674 .

(28) Xu, J.; Schwentner, N.; Chergui, M. J. Chem. Phys. 1994, 101, 7381.

(29) Kiviniemi, T.; Aumanen, J.; Myllyperkio, P.; Apkarian, V. A.; Pettersson, M. J. Chem. Phys. 2005, 123.

(30) Schofield, D. P.; Jordan, K. D. J. Phys. Chem. A 2007, 111, 7690.

(31) Geilhaupt, M.; Dorfmueller, T. Chem. Phys. 1983, 76, 443

(32) Ovchinnikov, M.; Apkarian, V. A.; Voth, G. A. J. Chem. Phys. 2001, 114, 7130 .

(33) Sloan, E. D.; Fleyfel, F. Fluid Phase Equilib. 1992, 76, 123.

(34) White, M. A.; MacLaren, D. C.; Marriott, R. A.; Zhan, B.-Z. Can. J. Phys. 2003, 81, 175

(35) Anderson, B. J.; Tester, J. W.; Borghi, G. P.; Trout, B. L. J. Am. Chem. Soc. 2005, 127, 17852.

(36) Hehre, W. J. A Guide to Molecular Mechanics and Quantum Chemical Calculations; Wavefunction: Irvine, CA, 2001.

(37) Spartan '04; Wavefunction Inc.: Irvine, CA, 2004.

(38) Kirchner, M. T.; Boese, R.; Billups, W. E.; Norman, L. R. J. Am Chem. Soc. 2004, 126, 9407.

(39) Saiz-Lopez, A.; Saunders, R. W.; Joseph, D. M.; Ashworth, S. H.; Plane, J. M. C. Atmos. Chem. Phys. 2004, 4, 1443. 